\title{
Typology of schools operating in the Moscow Electronic School system based on the analysis of network indicators
}

\author{
Svetlana Vachkova ${ }^{1 *}$,Elena Petryaeva ${ }^{1}$, and Evgeny Patarakin $^{1}$ \\ ${ }^{1}$ Moscow City University, Institute of System Projects, Moscow, Russia
}

\begin{abstract}
Network analysis methods are actively used to research the behavior of digital repository users who utilize and create digital objects. At the same time, the research into the collective behavior of a group of participants who are members of the same school is much less common. The library of the Moscow Electronic School is a rather complicated system with multiple roles offered to users. The actors of the repository are teachers, students, parents, and publishers - anyone performing any actions with the objects. In this study, the school is seen as an actor performing actions with objects - lesson scenarios within the Moscow Electronic School repository of digital objects. Within the study, the authors compare the sociograms of schools that unite teachers and the scenarios created by the teachers and divide schools into factions based on network indicators in sociograms. The main method of presenting and analyzing data is network analysis and sociogram creation. The authors identify two types of networks: the network of single participant's relationships and the network of relationships of teachers from a single school. The authors not only describe the data structure in the Moscow Electronic School system that records the digital trace of every individual and collective user but also create a digital map that reflects the dynamics of actions in the Moscow Electronic School system and identify the indicators that characterize the common activity of key participants. Moreover, the authors identify graph factions for schools that characterize the degree of interaction between teachers: disconnected groups, sparse graphs, crystallization centers, dense graphs.
\end{abstract}

Keywords: digital repository, interaction of teachers, network analysis, digital object.

\section{Introduction}

In recent years, the number and variety of learning object repositories (LOR) have increased. The actions of the creators and users of digital objects inside the repositories attract the attention of researchers who analyze both the behavior of individual participants and the formation of groups and communities of participants interacting based on the use of materials from the repositories [1-3].

\footnotetext{
*Corresponding author: svachkova@mgpu.ru
} 
Digital objects are usually defined as editable, interactive, open, duplicated, and distributed $[4,5]$. Reusability means that a digital object can be reused in a new learning context and does not need to be tied to the discipline or subject for which the object was created. With all the variety of digital objects, educational analysts always strive to find a social object that students exchange [6]. Social theories of collaboration are closely connected to modern network science, the application of which to situations of collaborative educational practice allows one to gain new knowledge necessary for making management decisions. The collaboration of learners in various collaborative environments (Wiki, Scratch, and others) is quite often the subject of study, support, and purposeful design [7, 8]. However, teachers' cooperation is studied much less often. Among the online platforms that support teachers' reusing educational practice scenarios, one should first mention the British community ClowdWorks [9], which, however, froze its activities in 2018 and currently operates as an archive of uneditable materials. Given this, the Moscow Electronic School (MES) project with the option to reuse lesson scenarios [10] is of considerable research interest.

\section{General scheme of users' operations inside digital repositories}

In the early 21 st century, the ability to reuse another person's work became widespread. Remix culture has had a significant impact on education and new educational strategies [8, $11,12]$.

With all the variety of forms of collabortive network activity in all systems, this type of activity can be represented as a single scheme that allows one to analyze and compare participants' activities. Various types of socio-technical systems, in which the collaborativeactivities of the participants are realized, save in the logs of all the participants' actions: subject (agent), object (article, program, scenario), type (creation, copying, downloading), and duration of the activity.

Various forms of collaborative network activity can be reduced to graphs: bipartite graphs, where subjects and objects of activity are represented, and unipartite 1 graphs of the participants' relationships [13-15].

Since there is a digital footprint of actions on shared digital objects, we can build a digital map of the shared actions of participants in the MES. As a result of highlighting and visualizing data for each teacher, we get a common data structure that captures the digital history of the teacher's relationship with other participants.

The invariance of actions makes it possible to obtain sociograms of relationships in various communities and determine the roles of community members based on network characteristics. In a work on the typology of roles in Wikipedia, the authors [16] used sociograms to highlight the participants in the general field of the project. Yang discusses the various social roles that can be computed [17]. There are two key challenges that community designers face: the quality of the content that members post and keeping members engaged in using and improving the content.

Henderson provides a typology of members based on their network characteristics: centers of stars and clique members [18]. This typology is built not on the actions performed by participants but on the form of groups and the position that individual participants hold in groups on the field of joint activity. We find Henderson's approach interesting since this approach is related to the theory of community of practice, for which the process of moving agents from the periphery to the center of the community through establishing connections with objects and agents of activity is important. 


\section{Methods}

All actions of the MES participants are recorded by the system in the log files of individual actions. For data processing, visualization, and statistical analysis, we used the R language, in particular, the approach of tidy data presentation [19]. As a result of combining log files for various actions of participants on objects, we obtained a table with more than 30 million actions by 60 thousand teachers on more than 40 thousand digital objects.

The table of actions provides a visual representation of the activities by all participants, individual schools, and specific teachers.

When creating a digital map reflecting the dynamics of the participants' joint actions, we based our actions on the basic Network Science approach. Network analysis and the creation of sociograms were chosen as the main methods of data analysis.

This technique was also used to identify groups of teachers from the same school. We identified numerous school groups based on the fact that the actions of each actor are performed by teachers from the same school, presented the history of each actor's actions in the form of a bipartite graph, and decomposed graphs into factions based on their network indicators.

When there are fewer than 100 nodes in a bimodal graph representing the relationship of teachers and lesson scenarios, the sociogram is quite simple to view and interpret. In Figure 1 , one can see that the actions of many teachers in the school are associated with a single scenario. Several more scenarios are located at the periphery of the graph and have attracted the attention of single participants.

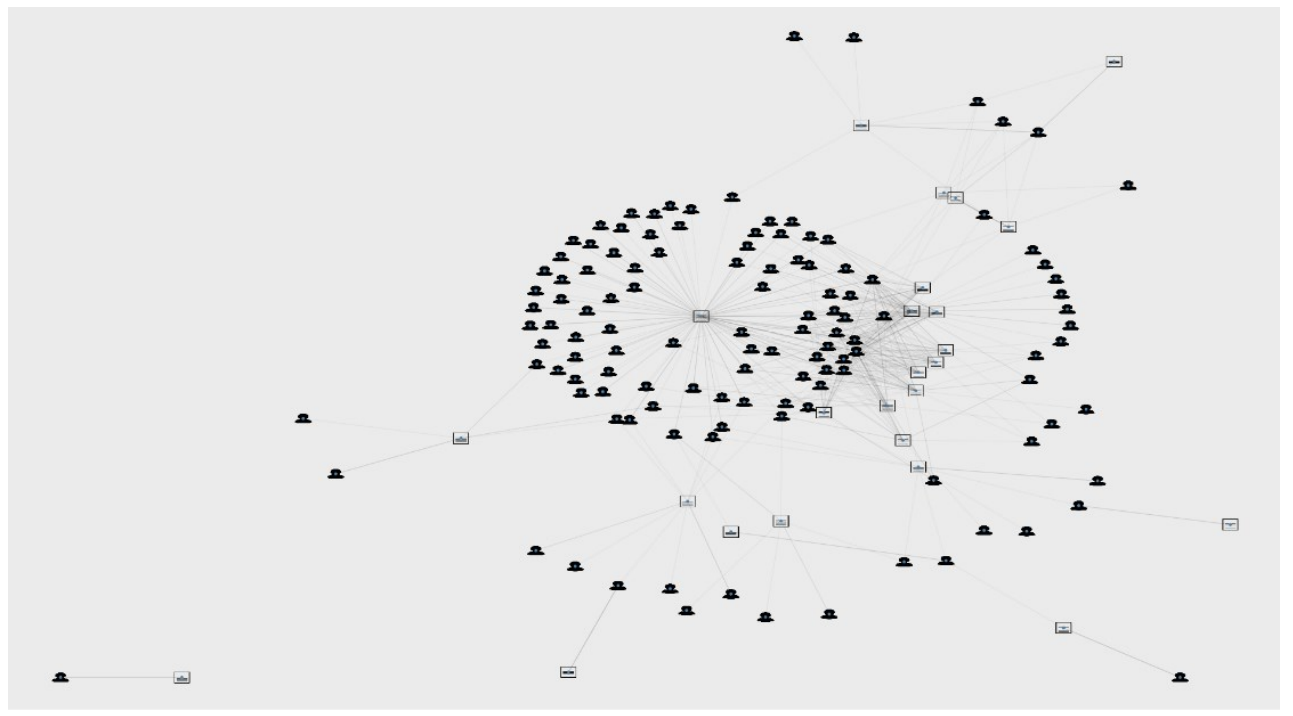

Fig. 1. Bipartite graph of a school.

If bipartite graphs of schools contain many participants and objects created by them, the situation can be simplified and converted to a unipartite 1 graph, in which only teachers connected with each other will be represented. A unipartite graph allows one to obtain an indicator of subject-directed interactions - the clustering coefficient. The clustering coefficient of a node is the probability that the two nearest neighbors of this node are also the nearest neighbors. The global clustering coefficient shows the level of connectivity and interaction of the group as a collective subject of activity. Connectivity is especially great in completely interconnected groups - cliques (Fig. 2). 


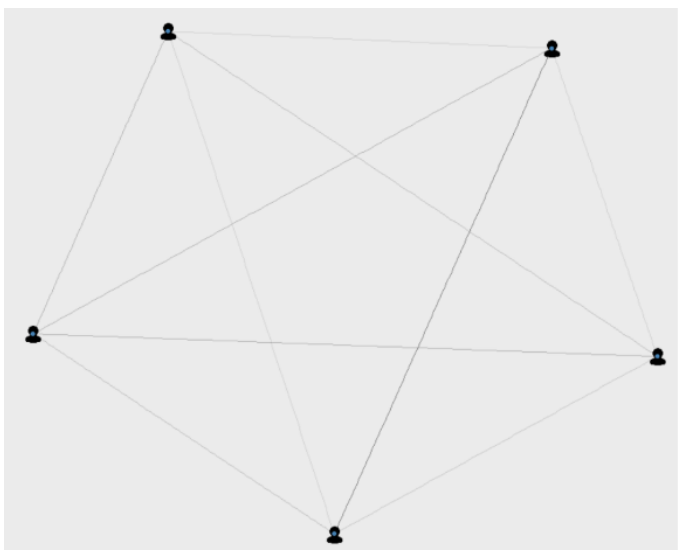

Fig. 2. A clique - a group of interconnected teachers.

\section{Results}

We distinguish four indicators characterizing the activity of schools in the MES system.

The number of participants is associated with a particular school within the MES.

Productivity is the ratio of the number of created objects to the number of participants in the activity.

Usability reflects the number of accesses to scenarios. This indicator characterizes not only the popularity of the published scenarios but also the activity of interaction between the participants in shared activities.

Density is determined by the number of connections between subjects and objects of collabortive activity as a result of subject-directed interactions. The value of the indicator can be used to judge the intensity of interaction between the actors of collaborative activities associated with the creation, viewing, copying, evaluation, and demonstration of scenarios. The density indicator can be applied to the network of links of an individual school, subject-based methodological association, or the entire network of the MES educational scenario repository. The density of a bipartite network characterizes the intensity of subject-directed interactions between participants.

The connectivity of the system is estimated by the number of independent components. The more such groups, the lower the system connectivity. The connectivity indicator is easy to obtain for bipartite and unipartite graphs. To assess the system of collaborative activity, in addition to this indicator, one can use the indicator of the number of components in unrelated groups in the graph of subjects and objects of collaborative activity. The greater the number of independent components, the lower the degree of connectivity and the more disconnected the participants in collaborative activities are.

Sociograms of schools can be viewed as structures in which the number of nodes (teachers and scenarios) and the density of connections that these nodes unite are gradually increasing. The history of most schools in the MES system begins with disconnected graphs. An example of a school in which teachers working in the MES are practically not connected to each other is shown in Figure 3. 


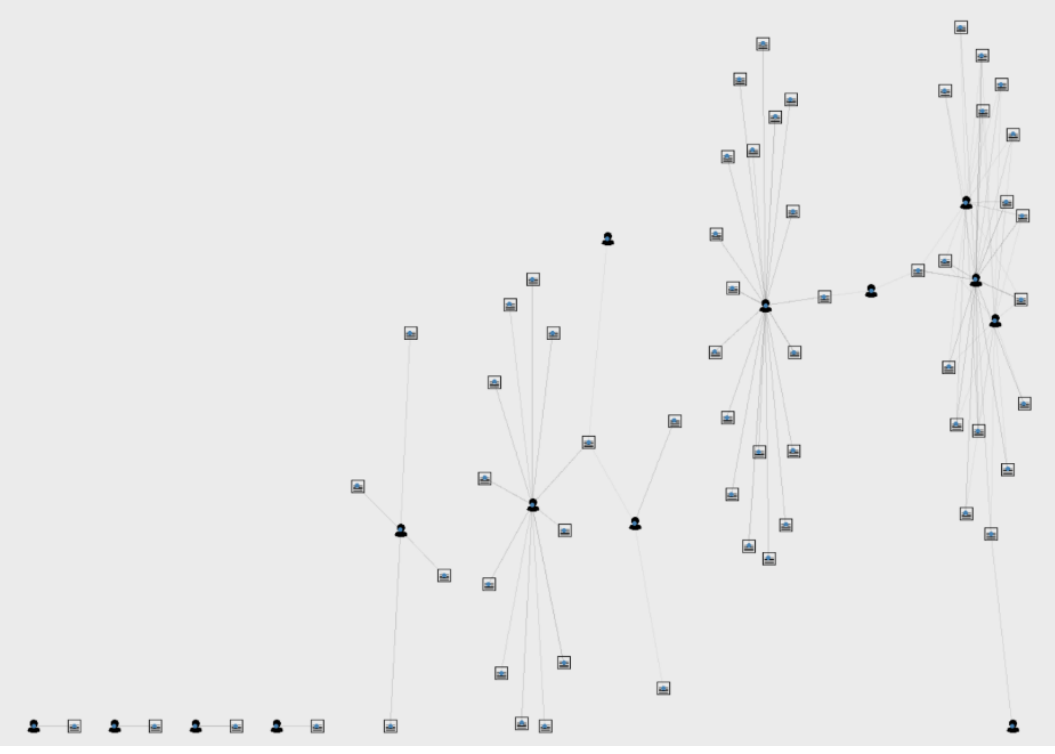

Fig. 3. A disconnected graph.

Gradually, new connections arise between the participants and the graphs turn from disconnected into sparse, where the number of nodes is greater than or equal to the number of connections. At the same time, most of the participants are located at the edge of the network, and most of the scenarios are associated only with the authors who published them. In Figure 4, on the periphery, there are school teachers who performed single actions with scenarios that were published by teachers from the same school.

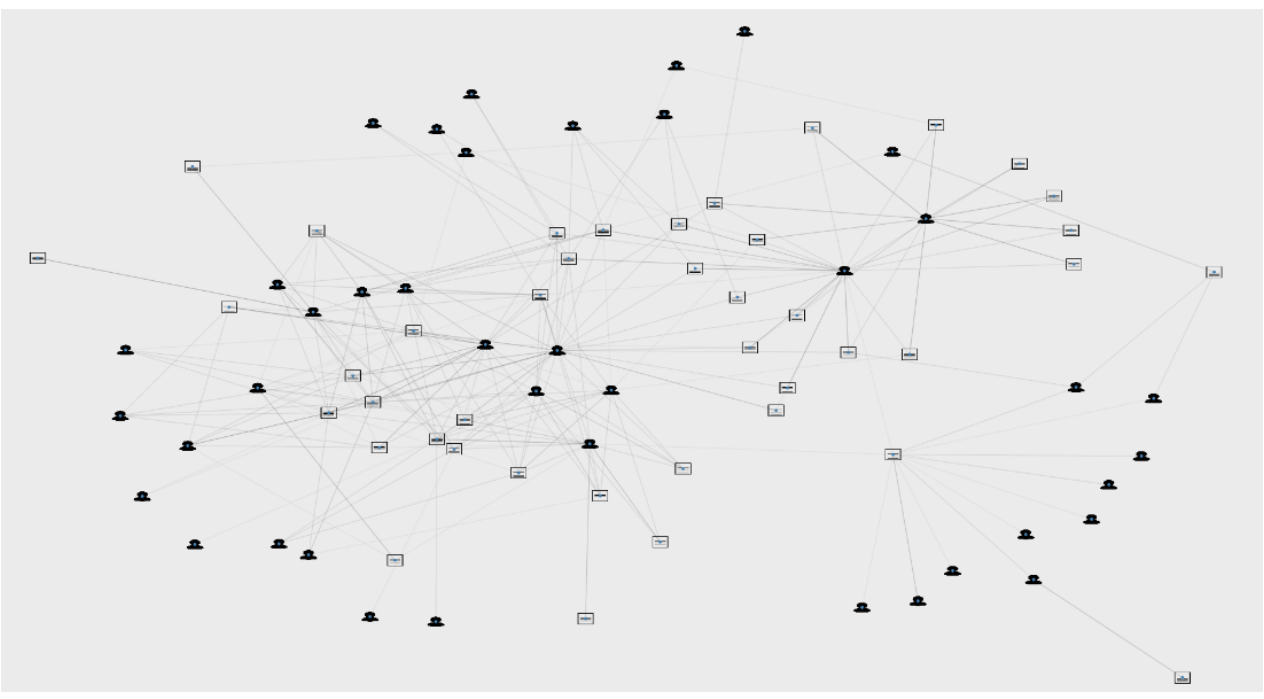

Fig. 4. A sparse graph.

Gradually, within the bipartite graph of the school, the number of connections between teachers and the utilized scenarios grows, and usually, several centers of crystallization appear. For example, in Figure 5 there is the graph of the school that shows the initial stage of the formation of teacher groups in the left and right parts of the graph. Moreover, the group on the left is much denser. 


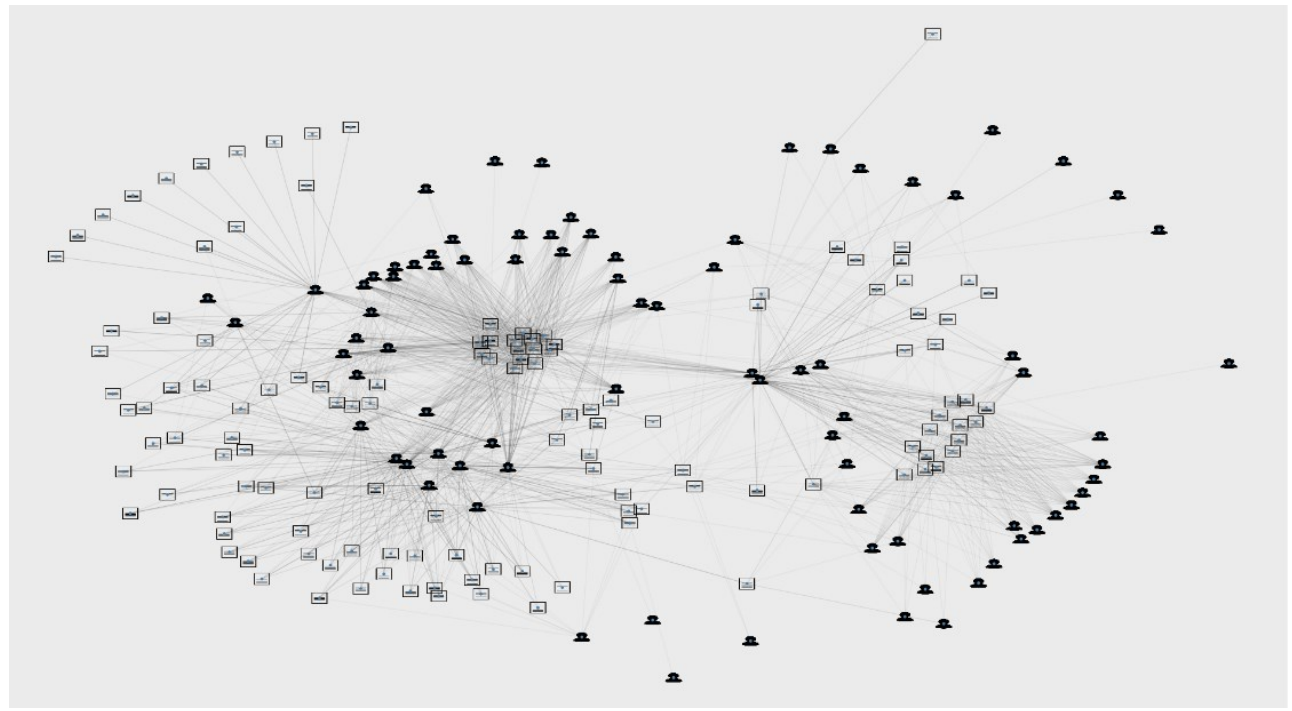

Fig. 5. Group crystallization centers.

Further development of the school network containing authors and users of MES scenarios can contribute to the selection of several distinguishable clusters in the graph. However, much more often one encounters dense graphs with a very high density of connections within one component, which all teachers of the school strive to join (Fig. 6).

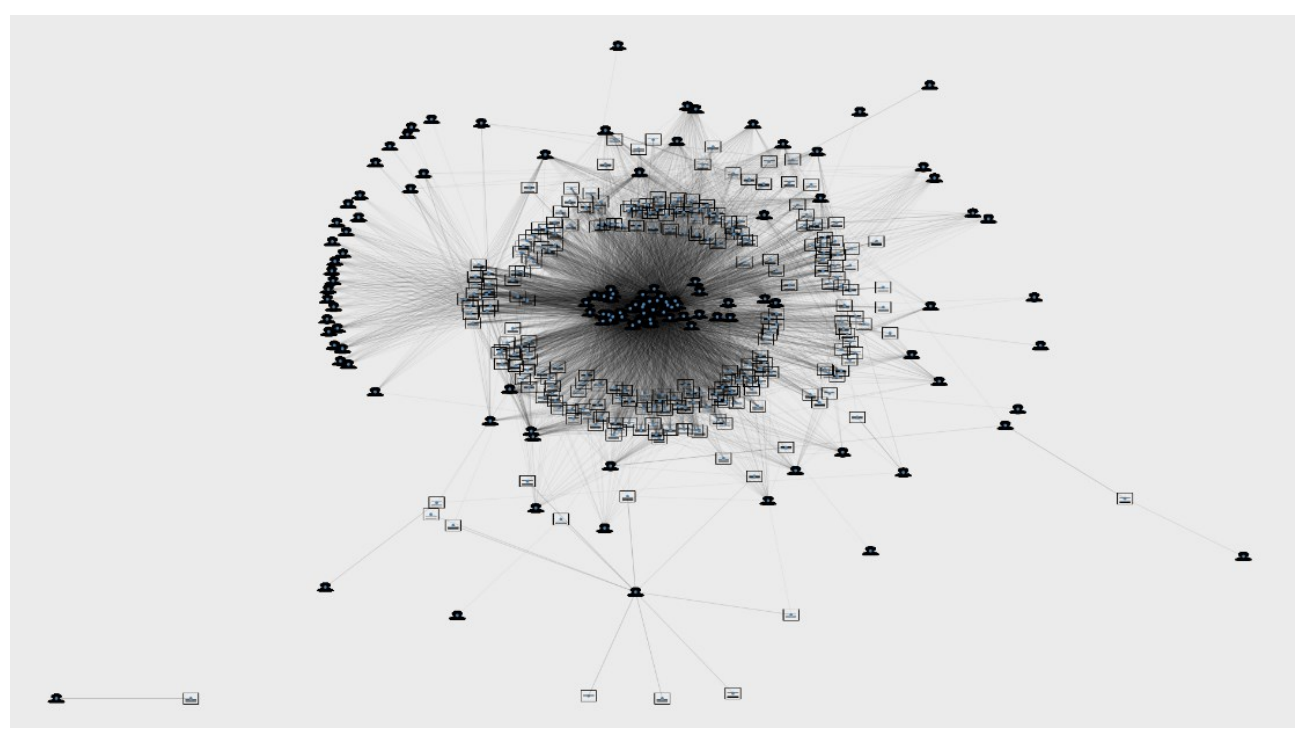

Fig. 6. A dense graph of the school.

\section{Conclusion}

Cases of using the MES and the structure of intra-school groups uniting teachers are analyzed. The data structure that records the digital footprint of an individual and collective participant is described, a digital map is created that reflects the dynamics of actions in the MES system, indicators are identified that characterize the joint activities of key participants. The connection between the actions of the participants and the emerging 
network social structure has been established. The study shows that a bimodal graph representing the connections between teachers and the lesson scenarios the teachers have created can serve as a convenient form of visualization that expands the possibilities for assessing and discussing the school as a single agent that fills and uses the digital educational repository.

\section{References}

1. A. Kumar, R. Saigal, R. Chavez, N. Schwertner, Architecting an extensible digital repository, in Proceedings of the 4th ACM/IEEE-CS Joint Conference on Digital Libraries, JCDL '04. ACM, New York, USA, 2-10 (2004). https://doi.org/10.1145/996350.996354

2. S. Wang, Interdisciplinary Journal of E-Learning and Learning Objects, 4, 1-12 (2008)

3. S. Yassine, S. Kadry, M.A. Sicilia, Learning Analytics and Learning Objects Repositories: Overview and Future Directions, in: Learning, Design, and Technology: An International Compendium of Theory, Research, Practice, and Policy (Springer International Publishing, Cham, 2016). https://doi.org/10.1007/978-3-319-17727-4_131

4. J. Kallinikos, A. Aaltonen, A. Marton, First Monday, 15(6), (2010). Accessed on: December 16, 2020. [Online]. Available: https://firstmonday.org/ojs/index.php/fm/article/view/3033/2564

5. A. Marton, J. Kallinikos, When Forgetting Becomes Digital: Social Memory in the Age of Digital Reproduction, Paper presented at The 33rd EGOS Colloquium 2017, Copenhagen Business School, 6-8 July, 2017, Copenhagen, Denmark (2017)

6. N. Hussain, H.H. Wang, C.D. Buckingham, X. Zhang, Int. J. Soft. Eng. Knowl. Eng., 30(06), 859-893 (2020). https://doi.org/10.1142/S0218194020400100

7. M. Barajas, F. Frossard, Educ Inf Technol, 23, 1403-1419 (2018). https://doi.org/10.1007/s10639-017-9674-2

8. S. Dasgupta, W. Hale, A. Monroy-Hernández, B.M. Hill, Remixing as a Pathway to Computational Thinking, in Proceedings of the 19th ACM Conference on ComputerSupported Cooperative Work \& Social Computing, CSCW '16. ACM, New York, USA, 1438-1449 (2016). https://doi.org/10.1145/2818048.2819984

9. G. Conole, IJOER, 1(1), (2018). https://doi.org/10.18278/ijoer.1.1.6

10. S.N. Vachkova, E.D. Patarakin, E.Y. Petryaeva, SHS Web Conference, 79, 01017 (2020). https://doi.org/10.1051/shsconf/20207901017

11. C. Fiesler, A.S. Bruckman, Remixers' Understandings of Fair Use Online, in Proceedings of the 17th ACM Conference on Computer Supported Cooperative Work \& Social Computing, CSCW '14, SIGCHI, February 2014, Baltimore Maryland, USA (2014). https://doi.org/10.1145/2531602.2531695

12. O. Seneviratne, A. Monroy-Hernandez, Remix culture on the web: A survey of content reuse on different User-Generated content websites (poster paper), in Proceedings of Web Science Conference (Web Sci '10) (2010). Accessed on: December 16, 2020. [Online]. Available: https://www.media.mit.edu/publications/remix-culture-on-theweb-a-survey-of-content-reuse-on-different-user-generated-content-websites/

13. E. Patarakin, V. Burov, Using of Automatically and Semi-automatically Generated Diagrams in Educational Practice, in Electronic Governance and Open Society: Challenges in Eurasia. EGOSE 2019. Communications in Computer and Information 
Science, 13-14 November 2019, Saint Petersburg, Russia (2020). https://doi.org/10.1007/978-3-030-39296-3_23

14. E. Patarakin, V. Burov, R. Parfenov, Identifying sets of key players and cliques in socio-educational co-creative projects, in Proceedings of the 2015 Conference on Electronic Governance and Open Society: Challenges in Eurasia, EGOSE '15, November 2019, Saint Petersburg, Russia (2015). https://doi.org/10.1145/2846012.2846028

15. E.D. Patarakin, Wikigrams-Based Social Inquiry, in Digital Tools and Solutions for Inquiry-Based STEM Learning, 112-138, (IGI Global, Hershey, 2017). https://doi.org/10.4018/978-1-5225-2525-7.ch005

16. H.T. Welser, D. Cosley, G. Kossinets, A. Lin, F. Dokshin, G. Gay, M. Smith, Finding social roles in Wikipedia, in Proceedings of the 2011 IConference, IConference '11. Association for Computing Machinery, February 2011, Seattle, Washington, USA (2011). https://doi.org/10.1145/1940761.1940778

17. D. Yang, Computational Social Roles, Thesis (Carnegie Mellon University, Pittsburgh, 2019). Accessed on: December 30, 2020. [Online]. Available: https://www.lti.cs.cmu.edu/sites/default/files/yang\%2C\%20diyi.pdf

18. K. Henderson, B. Gallagher, T. Eliassi-Rad, H. Tong, S. Basu, L. Akoglu, D. Koutra, C. Faloutsos, L. Li, RolX: structural role extraction \& mining in large graphs, in Proceedings of the 18th ACM SIGKDD International Conference on Knowledge Discovery and Data Mining, KDD '12, Association for Computing Machinery, 12-16 August, Beijing, China (2012)

19. H. Wickham, G. Grolemund, R for Data Science: Import, Tidy, Transform, Visualize, and Model Data (O'Reilly Media, Sebastopol, CA, 2016) 